\title{
DELINEAMENTO DE COPAS DE ÁRVORES EM FOTOGRAFIAS AÉREAS DE ALTA RESOLUÇÃO, ATRAVÉS DE SUAS SOMBRAS PERIFÉRICAS: ESTUDO DE CASO NA FLORESTA OMBRÓFILA MISTA ${ }^{1}$
}

\author{
Attilio Antonio Disperati ${ }^{2}$ e Paulo Costa de Oliveira Filho ${ }^{2}$
}

\begin{abstract}
RESUMO - O presente artigo trata do delineamento semi-automático de copas de árvores através de suas sombras periféricas observadas em fotografia aérea de alta resolução. O estudo foi realizado em área de dois hectares de Floresta Ombrófila Mista da Floresta Nacional de Irati, PR, utilizando-se a técnica de interpretação, nos formatos analógico e digital, em uma fotografia aérea de $70 \mathrm{~mm}$ colorida normal, na escala de 1:1.000, e dados obtidos em campo. A metodologia consistiu na transformação das três bandas originais da fotografia colorida digital em apenas uma e na separação da componente sombra dos demais alvos restantes da imagem, através de um limiar. A partir dos resultados, verificou-se que: a) as sombras podem ser visualizadas em clareiras existentes na floresta, entre os galhos e entre as copas das árvores; b) a imagem resultante evidencia a inconsistência de se mapearem as copas exclusivamente pelas sombras periféricas; c) o resultado insatisfatório foi atribuído ao tipo de floresta natural considerado; d) a técnica mostrou-se promissora como ferramenta adicional de realce de imagem; e e) faz-se necessário desenvolver maior número de pesquisas para se obterem resultados conclusivos.
\end{abstract}

Palavras-chave: Fotografia aérea de alta resolução, imagem iluminação/albedo, sombras periféricas e Floresta Ombrófila Mista.

\section{DELINEATION OF INDIVIDUAL TREES IN HIGH RESOLUTION AERIAL PHOTOGRAPHS, THROUGH THEIR PERIPHERAL SHADOWS: CASE STUDY IN ARAUCARIA FOREST}

\begin{abstract}
This paper deals with the semi automatic delineation of crowns of trees on high resolution aerial photograph using their peripheral shadows. The study was carried out on 2 hectares of native Araucaria forest due to the avaliability of field data and the interpretation, in analogic and digital formats, of a 70 mm 1:1.000 high resolution aerial photograh. The methodology consisted in the transformation of the 3 original bands of the digital color photograph in only one, and then, through a threshold, the shadow component was separated from the other targets. From the results obtained, the main conclusions reached were: a) the shadows were due to the open areas on the forest among the branches and the crowns of the trees; $b$ ) the resultant image evidenced the inconsistency to delineate the crowns exclusively through their peripheral shadows; c) the inadequate result may be related to the type of natural forest and not to the technique itself; d) the used technique showed promising as an additional enhancement technique, and e) it is necessary to carry out more research in order to obtain conclusive results.
\end{abstract}

Key words: high resolution aerial photograph, illumination /albedo image, peripheral shadows, araucaria forest.

\footnotetext{
${ }^{1}$ Recebido em 28.04.2004 e aceito para publicação em 25.11.2004.

${ }^{2}$ Departamento de Engenharia Florestal da Universidade Estadual do Centro-Oeste - UNICENTRO, BR 153 - km 7 - Riozinho/ Irati-PR - 84500-000 - Caixa Postal 21. E-mail:<disperati@avalon.sul.com.br>.
} 


\section{INTRODUÇÃO}

A partir da década de 1990, apoiado nos benefícios das geotecnologias, na disponibilidade crescente de softwares de processamento digital de imagens e reconhecimento de padrões e no desenvolvimento de hardwares e de novos produtos de sensores remotos de alta resolução, surgiu a linha de pesquisa denominada "Interpretação automática de imagens digitais de alta resolução para a atividade florestal" (Automated interpretation of high spatial resolution digital imagery for forestry) (BRANDTBERG, 2001).

A finalidade básica desta linha de pesquisa é disponibilizar informações suficientes para que o processamento e análise digital de imagens sejam automatizados, minimizando a intervenção humana no processo. Embora ainda no estágio inicial, ela se mostra bastante promissora em termos de desenvolvimento de rotinas específicas para essa finalidade nos sistemas de análise de imagens de alta resolução.

O termo imagem engloba diversos produtos de sensores remotos, a saber: fotografias aéreas digitais (provenientes de câmaras digitais ou da digitalização da fotografia analógica); imagens satelitárias (QUICKBIRD, IKONOS, SPOT 5) e imagens de sensores multiespectrais instalados a bordo de aeronaves (CASI, MEIS etc.). Enquanto para Quackenbush et al. (2000) a resolução espacial desse tipo de imagem é menor do que $5 \mathrm{~m}$, para Gougeon (1993) está compreendida entre 10 e $100 \mathrm{~cm}$. Apesar desse aspecto contraditório, a implicância do tamanho individual do pixel na imagem digital é fato sobejamente conhecido. Nesse sentido, Leckie (1992) comentou que, em imagens com resolução espacial de 0,72 m, é possível observar as árvores individuais; com 1,5 m, começam-se a perder detalhes; e com 3 a 6 m, as árvores não são distinguíveis.

A imagem de alta resolução, com acentuada definição espacial, permite observar a parte superior da árvore em detalhes, incluindo suas copas, galhos, partes sombreadas entre os galhos etc. Segundo Gougeon (1999), deve-se "pensar" em árvores individuais ao invés de em talhão florestal, sendo factível o traçado ou o delineamento das respectivas copas.

Nas décadas de 1950 a 1970, o delineamento do perímetro das copas das árvores foi intensamente utilizado em trabalhos para obter dados de diâmetro e área da copa da árvore, densidade por hectare e posterior

R. Árvore, Viçosa-MG, v.29, n.2, p.195-202, 2005 regressão estatística para determinar o DAP da árvore em função do diâmetro de copa (OLIVEIRA, 1980), assim como elaborar tabela de volume aérea (STELLINGWERF, 1973).

Segundo Disperati e Oliveira-Filho (2002) apud Brandtberg (2001), os três métodos fundamentais para delinear ou localizar copas individuais de árvores em imagens digitais de alta resolução são: detecção do local de intensidade máxima ("detection of local intensity maximum"), baseado em contorno ("contour-based methods"), e comparação de padrões de gabarito ("template-based matching"). Não é possível comparar os métodos entre si, pois eles utilizam diferentes princípios baseados nos níveis de informações das imagens.

O primeiro método determina a posição espacial da ponteira do fuste da árvore na imagem digital, pois ela geralmente corresponde ao ponto de máxima intensidade. Esse ponto é determinado através de uma janela (dimensão do filtro) de varredura um pouco maior do que o tamanho da copa da árvore na imagem; em seguida, o delineamento da copa da árvore na imagem pode ser feita através das sombras periféricas situadas em torno do ponto de máxima intensidade; o segundo determina e utiliza basicamente as partes ou áreas sombreadas entre as copas das árvores e, através delas, por rotinas específicas de computador, traça os limites das copas; e o terceiro dispensa a consideração de áreas sombreadas entre as copas por utilizar padrões de gabarito de copas específicos para cada espécie florestal considerada. A aplicação desses métodos que utilizam rotinas ou softwares de computador desenvolvidos especificamente para a tarefa proposta tem mostrado que os melhores resultados são obtidos quando se processam imagens de plantios de coníferas, visto que o formato cônico das árvores resulta em um padrão na imagem, que facilita a determinação da ponteira do fuste da árvore (através do ponto de intensidade máxima) e o respectivo delineamento da copa da árvore (através dos limites das áreas sombreadas). Porém, em floresta natural, o entrelaçamento das copas das árvores de diversas espécies, às vezes compondo vários estratos arbóreos de diferentes tamanhos (em altura e copa) e espaçamento variável, dificulta a caracterização de copas individuais nas imagens aéreas.

Apesar da escassez de aplicações dessa nova técnica em floresta natural, Warner et al. (1999), analisando fotografias aéreas de alta resolução de uma área de 
floresta decidual localizada no leste dos Estados Unidos, verificaram que o método mais simples para identificar o contorno da copa das árvores é aquele que utiliza as sombras periféricas de cada árvore. A metodologia adotada pelo mesmo autor foi baseada na "transformação hiperesférica por co-senos direcionais" (hyperspherical direction cosine transformation), proposta por Pouch e Campagna (1990), e consiste na criação de uma nova imagem a partir das três bandas de uma fotografia aérea colorida, denominada imagem iluminação/albedo. Ela combina ambas as variações causadas pela iluminação e pela reflectância geral presente nas três bandas e é obtida através do cálculo da raiz quadrada do somatório do quadrado dos pixels individuais das bandas consideradas. Em seguida, utilizando um limiar de determinado valor digital, obtido subjetivamente através da análise da imagem iluminação/albedo, efetua-se a separação da componente sombra dos alvos restantes da imagem. O método é simples e não necessita de um algoritmo sofisticado.

A finalidade da presente pesquisa é aplicar o mesmo procedimento utilizado por Warner (1999) para delinear as copas de árvores, localizadas em um experimento em uma área natural de Floresta Ombrófila Mista (FOM) situada na Floresta Nacional (Flona) de Irati, PR, a partir de fotografia aérea $70 \mathrm{~mm}$ colorido normal em escala 1:1.000 e formato digital.

\section{MATERIAL E MÉTODOS}

\section{1. Área de estudo}

A área de estudo abrange 2 ha de um experimento de 25 ha de Floresta Ombrófila Mista (FOM), protegida por mais de 50 anos, situada na Floresta Nacional (Flona) de Irati, unidade de uso sustentável do IBAMA, distante, aproximadamente, $150 \mathrm{~km}$ a oeste de Curitiba, no Estado do Paraná. A área experimental (25 ha) instalada em 2001 para estudos multidisciplinares é composta de 25 blocos de 1 ha, sendo cada bloco formado por quatro parcelas de 50 x 50 m (DISPERATI, 2002a).

Uma análise detalhada de reconhecimento e identificação das copas de árvores foi conduzida nos dois primeiros hectares do experimento (parcelas 1, 2, 3 e 4 do bloco 1; parcelas 1 e 3 do bloco 2; e parcelas 3 e 4 do bloco 24). O trabalho de campo que permitiu a elaboração do mapa de copas de cada parcela dos blocos, mostrando a posição espacial das copas das árvores dos estratos superiores e sua relação com as demais copas vizinhas, facilitou em muito o trabalho de fotointerpretação e possibilitou numerar cada copa, baseado na respectiva árvore no terreno, delineada visualmente no monitor do computador. Atenção especial foi dada para o delineamento individual e em grupo das copas de pinheiro-do-paraná, devido à sua dominância no estrato superior, e para as demais copas perfeitamente possíveis de serem caracterizadas, visto o detalhe da imagem e dos mapas de copas. Durante a interpretação, intenso trabalho de campo foi conduzido visando dirimir as dúvidas e dificuldades existentes diante do confronto da fotointerpretação com o mapa de copas e posição das árvores nos blocos. Após detalhada depuração da fotointerpretação, foram delimitadas, visualmente, 166 copas. Desse total, 12 espécies florestais (15\% das árvores mapeadas no terreno) estavam representadas por mais de quatro indivíduos, sendo as de maior freqüência o pinheiro-do-paraná (Araucaria angustifolia), com 54 copas (cujas copas nos 2 ha cobriam $34,8 \%$ da área) e as imbúias (Ocotea spp), com 29 (DISPERATI, 2002b; DISPERATI et al., 2002).

O número médio de árvores por hectare nas parcelas consideradas, com DAP (diâmetro à altura do peito) acima de $30 \mathrm{~cm}$, foi de 555 . A altura dos exemplares adultos de Araucária e com copas dominantes no dossel da floresta foi superior a $30 \mathrm{~m}$. Pelo porte em altura e em DAP das maiores árvores existentes, estima-se a idade da floresta como sendo superior a 300 anos. As coordenadas planas (Sistema UTM) centrais aproximadas dos 2 ha de estudo são: Fuso 22, N=7.193.000 e $\mathrm{E}=542.2000$. A altitude aproximada do local é de $860 \mathrm{~m}$, sendo o relevo, na sua maior parte, levemente ondulado.

\subsection{Material}

Foram utilizados os seguintes materiais:

a) Fotografia aérea não-métrica $70 \mathrm{~mm}$ colorida normal, ampliada para a escala 1:1.000, obtida no dia 8 de agosto de 2001, abrangendo parte da área experimental e digitalizada através do Scanner HP Jetscan 3400 com 300 dpi de resolução, resultando em um pixel de tamanho individual de aproximadamente $8 \mathrm{~cm}$, em um arquivo digital de 2391 x 3274 pixels (Figura 1).

b) Software Spring versão 4.0.

c) Arquivo vetorial da localização dos diversos blocos e do delineamento e numeração (idêntica à adotada no campo) das 166 copas de árvores (Figura 2a) consideradas nos 2 ha (DISPERATI, 2002b; DISPERATI et al., 2002).

R. Árvore, Viçosa-MG, v.29, n.2, p.195-202, 2005 

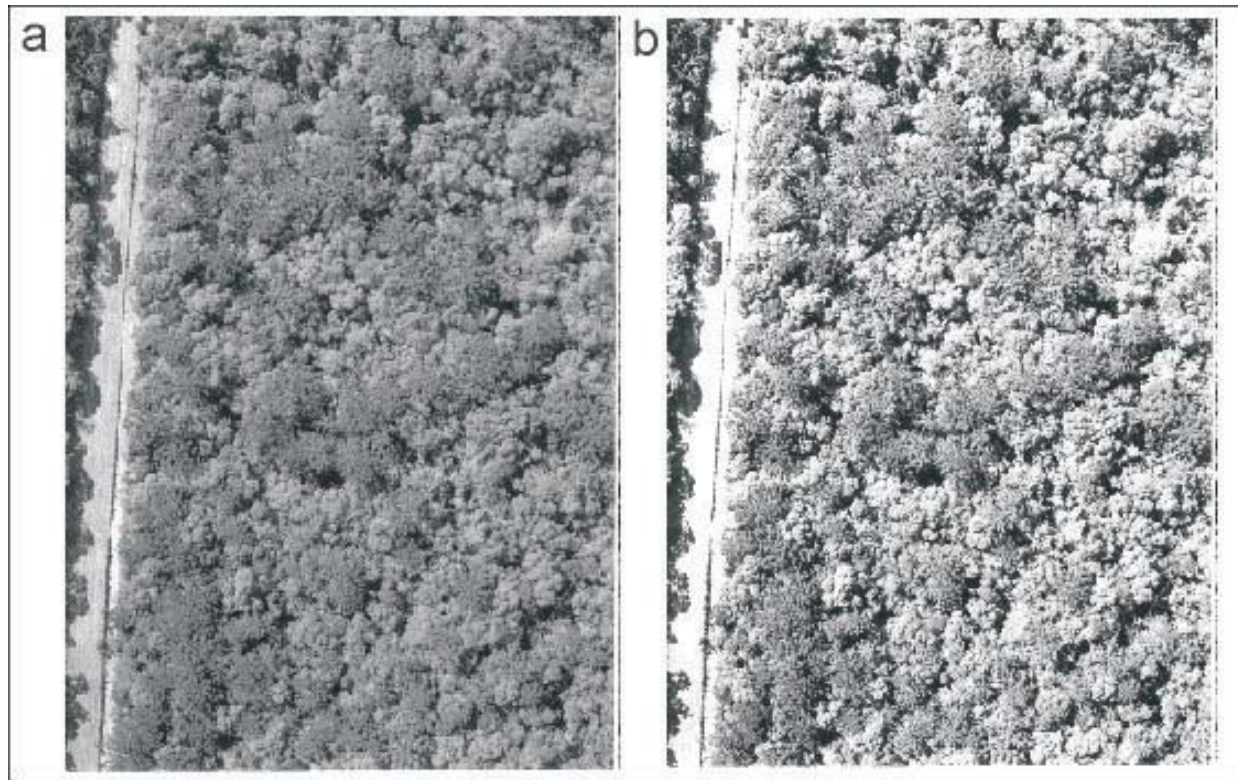

Figura 1 - Fotografia aérea colorida normal (a) e fotografia realçada (b).

Figure 1-Normal color aerial photograph (a) and enhanced photograph (b).

\subsection{Métodos}

A seqüência das atividades utilizadas constou de:

a) Aplicação de rotina (algoritmo), escrita em linguagem LEGAL (Linguagem Espaço-Geográfica Baseada em Álgebra), para gerar uma nova banda individual (imagem iluminação/albedo) a partir dos valores individuais dos pixels das três bandas $(R, G, B)$ da fotografia aérea colorida normal. A fórmula utilizada para gerar a nova imagem foi:

$$
M R=\sqrt{\sum\left(R^{2}+G^{2}+B^{2}\right)}
$$

sendo:

$M R=$ matriz resultante;

$R=$ matriz com os valores digitais da banda $\mathrm{R}$;

$G=$ matriz com os valores digitais da banda $\mathrm{G}$; e

$B=$ matriz com os valores digitais da banda B.

A rotina em LEGAL está descrita a seguir:

\{Imagem R, G, B, result ("nome da categoria onde esta a foto"), em que:

$\mathrm{R}=$ recupere (Nome="nome do PI");

R. Árvore, Viçosa-MG, v.29, n.2, p.195-202, 2005

$$
\begin{aligned}
& \mathrm{G}=\text { recupere }(\text { Nome="nome do PI"); } \\
& \mathrm{B}=\text { recupere (Nome="nome do PI"); } \\
& \text { result }=\text { Novo (Nome="nome do novo }
\end{aligned}
$$
PI',ResX=30,Res Y=30); //ResX e ResY, indica o tamanho do pixel da foto ou resolução; e

result $\left.=\operatorname{sqrt}\left(\mathrm{R}^{\wedge} 2+\mathrm{G}^{\wedge} 2+\mathrm{B}^{\wedge} 2\right) ;\right\}$.

b) Seleção e estatística de pixels representativas das áreas de sombras e de outras classes de alvos existentes na imagem digital, a fim de subsidiar o fatiamento da imagem em duas classes distintas: áreas sombreadas e os restantes alvos existentes. Esses pontos foram selecionados no contexto de toda a fotografia aérea, mas tendo como base a imagem realçada pela técnica de equalização do histograma, que se mostrava muito mais informativa do que a original.

c) Comparação visual dos resultados obtidos com o mapa evidenciando o delineamento das 166 copas de árvores nos 2 ha.

\section{RESULTADOS E DISCUSSÃO}

A imagem iluminação/albedo, resultante da aplicação da rotina de computador sobre a fotografia aérea, é mostrada na Figura $2 b$. 

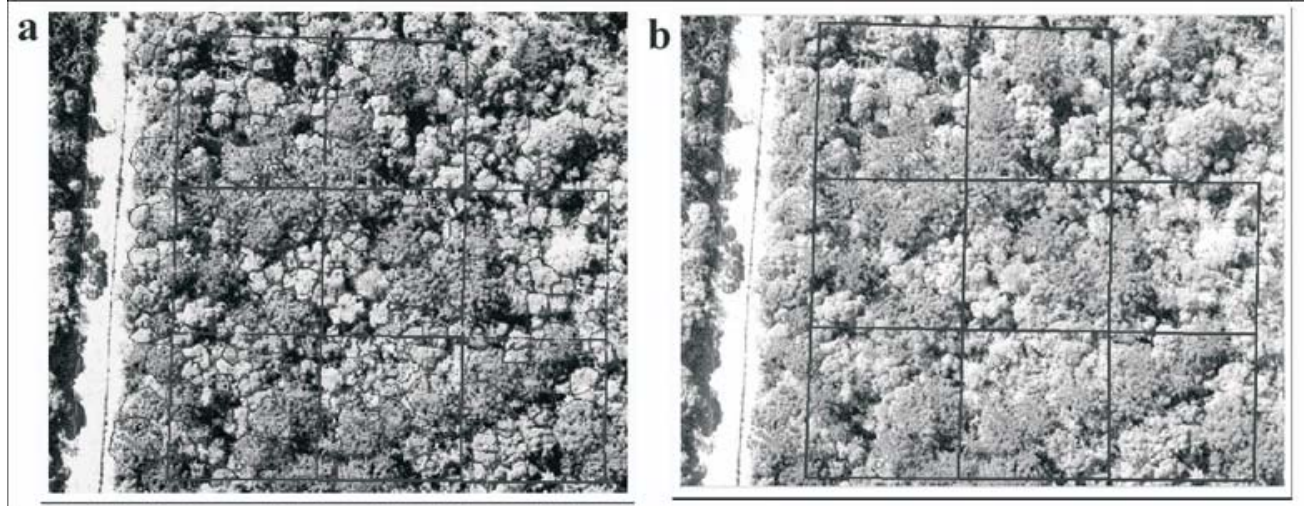

Figura 2 - Área do estudo (2 ha) mostrando o resultado da fotointerpretação (a) e a respectiva imagem iluminação/albedo (b).

Figure 2 -Study area (2 ha) with the photointerpretation results (a) and respective illumination albedo image (b).

A análise detalhada da imagem iluminação/albedo evidenciou a existência de áreas sombreadas em clareiras da floresta, entre as copas de árvores e entre os galhos de algumas copas de árvores.

A fim de verificar a possibilidade de separar visualmente as três classes na imagem através da técnica de fatiamento, diversos pixels foram amostrados para cada uma delas consideradas no contexto da imagem inteira. Na Tabela 1, mostram-se as estatísticas dos valores numéricos dos pixels referentes às três classes consideradas.

Os valores da Tabela 1 indicam certa similaridade de valores entre as classes sombra entre copas e sombra entre galhos. Apesar de os valores dessas duas classes aparentemente serem diferentes da classe sombra no terreno, há um recobrimento muito grande entre os seus valores digitais. Isso explica o motivo pelo qual não se obtiveram bons resultados por ocasião do fatiamento da imagem em quatro classes: 1) sombra

Tabela 1 - Estatística dos pixels das diversas classes consideradas na imagem iluminação/albedo

Table 1 -Statistics of the classes considered on the illumination/ albedo image

\begin{tabular}{lcccc}
\hline Classe & \multicolumn{3}{c}{ Valor digital } & $\mathrm{N}^{\circ}$ de \\
\cline { 2 - 4 } & Mín & Máx & Média & pixels \\
\hline Sombra no terreno & 45 & 137 & 98,5 & 47 \\
Sombra entre copas & 0 & 89 & 36,2 & 103 \\
Sombras entre galhos & 9 & 97 & 49,3 & 60 \\
\hline
\end{tabular}

entre copas, 2) sombra entre galhos, 3) sombras no terreno e 4) demais alvos na imagem. Diversas outras tentativas foram feitas englobando as outras duas classes. Apesar de elas apresentarem valores médios diferentes das classes referentes às áreas sombreadas, entre elas os resultados de separação, não foram adequados. Em face dessas análises preliminares conduzidas na imagem transformada, considerou-se apenas uma classe de sombra e partiu-se para a melhor definição do limiar para fatiar a imagem em apenas duas classes. Foram testados vários limiares, porém, pelos testes conduzidos, o melhor resultado subjetivamente considerado foi com o limiar 125. Abaixo desse valor digital, as áreas sombreadas eram poucas e acima desse valor, muitas. Assim, a imagem foi fatiada em duas classes (ver Figura 3): até o valor digital 125 representando em cor preta as sombras em torno das copas e após 125, representando os demais alvos existentes na imagem.

O valor digital 125, limiar utilizado no fatiamento da imagem, além dos pixels das classes sombra entre copas e sombra entre galhos, engloba parte dos pixels considerados na classe sombra no terreno, fato que vem complementar a análise da Tabela 1 .

A análise detalhada da imagem binária (Figura 3) indicou que somente a caracterização das sombras periféricas não foi suficiente para delinear, total ou parcialmente, as copas das árvores. As áreas sombreadas delimitadas na imagem iluminação/albedo mostraramse muito parecidas com as que poderiam ser delimitadas

R. Árvore, Viçosa-MG, v.29, n.2, p.195-202, 2005 
na imagem original e na imagem realçada, evidenciando que a técnica pode ser usada em complemento à análise visual. $\mathrm{O}$ analista da imagem terá mais facilidade de delinear visualmente o perímetro da copa da árvore quando observar as imagens original, realçada e a iluminação/albedo, acopladas em telas diferentes utilizando os recursos de acoplamento presentes em softwares de processamento de imagens.

A ineficiência das áreas sombreadas para delinear as copas deve-se ao tipo de floresta considerada, na qual existem pelo menos três estratos arbóreos verticais (dominante, co-dominante e intermediário), quando observado em fotografias aéreas, além de conter dezenas de espécies arbóreas com diferentes copas, tanto em formato quanto em tamanho. Esses aspectos da Floresta Ombrófila Mista, além de dificultarem a caracterização das copas individuais das árvores, não garantem o princípio de que as áreas sombreadas em torno das copas favorecem o delineamento da respectiva copa da árvore. Isso comprova o fato de que a aplicação dos métodos semi-automáticos e automáticos de delineamento individual de copas de árvores fornece melhores resultados em coníferas, onde o formato cônico das árvores e a distância entre elas resultam em um padrão bem visível e homogêneo.

O resultado, inferior ao obtido por Warner (1999), devido provavelmente ao fato de que, na imagem utilizada

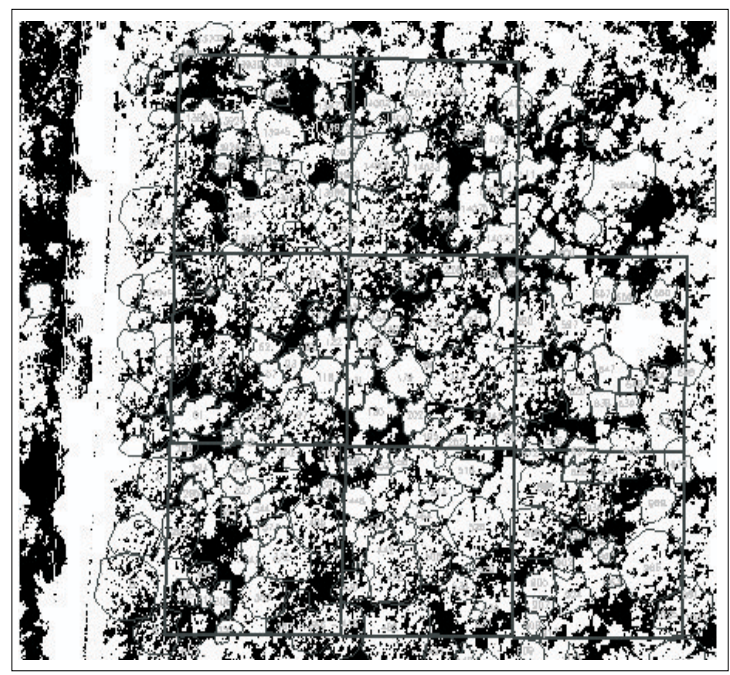

Figura 3 - Imagem binária resultante do fatiamento. Figure 3 -Binary (two levels) image of the study area. pelo pesquisador, muitas das copas das árvores não se tocavam, o que favorecia o delineamento das copas pelas áreas sombreadas.

A análise visual de algumas copas das espécies Araucaria angustifolia e Ocotea spp indicou que a maioria delas (ver Figura 4) não pode ser delimitada apenas pelas suas sombras periféricas.

A copa da Araucária, vista em uma fotografia aérea vertical, apresenta formato circular para elíptico, a qual, associada à disposição radial de seus galhos, resulta em um padrão característico. No entanto, os resultados mostrados nas imagens binárias (Figura 4) não ratificam com fidelidade o padrão característico da copa, dificultando o estabelecimento de um padrão de reconhecimento semi-automático. Em relação às imbúias, não ficou evidenciada uma forma típica de suas copas, porém, em comparação com as copas de Araucária, a quantidade de sombras internas é bem menor.

No manejo florestal, as áreas sombreadas podem facilitar os trabalhos de análise interpretativa da composição de espécies da floresta, sobretudo do dossel superior, principalmente com o uso da automatização desses processos interpretativos, utilizando-se técnicas de reconhecimento de padrões de copas. Áreas de sombras maiores na imagem indicam indícios de clareiras, cuja dinâmica, segundo Martins (2003), apresenta aplicações na silvicultura e no manejo de florestas nativas.

O fato de os resultados não serem conclusivos requer a realização de novos testes no contexto da FOM, particularmente a análise em florestas com menor densidade de árvores e de extratos arbóreos e, ou, com a presença uniforme de copas individuais, como povoamentos de bracatinga (Mimosa scabrella) e outras associações puras.

\section{CONCLUSÕES}

A partir dos resultados, chegou-se às seguintes conclusões:

- A fórmula utilizada para gerar a imagem iluminação/ albedo é simples e facilmente efetuada através de programação.

- A existência de áreas sombreadas em clareiras na floresta, entre as copas de árvores e entre os galhos das copas das árvores, não foi caracterizada no processo de fatiamento da imagem iluminação/albedo. 
- Os resultados insatisfatórios obtidos se devem ao tipo de floresta considerada e não à técnica em si.

- O uso da técnica não conduziu a um reconhecimento conclusivo do padrão típico das copas de Araucária e nem das imbúias, porém, nestas, a quantidade de sombras internas foi bem menor.

- A imagem transformada iluminação/albedo pode ser considerada como ferramenta adicional de realce de imagem.

- É necessário efetuar maior número de pesquisas para se chegar a resultados mais conclusivos e confiáveis.

\section{AGRADECIMENTOS}

Ao Dr. Timothy A. Warner, do Departamento de Geologia e Geografia da West Virginia University, Morgantown, WV, nos Estados Unidos, pelas informações adicionais de sua pesquisa.

Aoprogramador do INPELuigi Carli Marroni Aulicino, pela elaboração da rotina escrita em linguagem LEGAL.

Ao CNPq, pelo auxílio financeiro que possibilitou o desenvolvimento do projeto Biodiversidade $e$ Sustentabilidade da Floresta Ombrófila Mista na Flona de Irati, $P R$, cujos dados parciais foram utilizados nesta pesquisa.

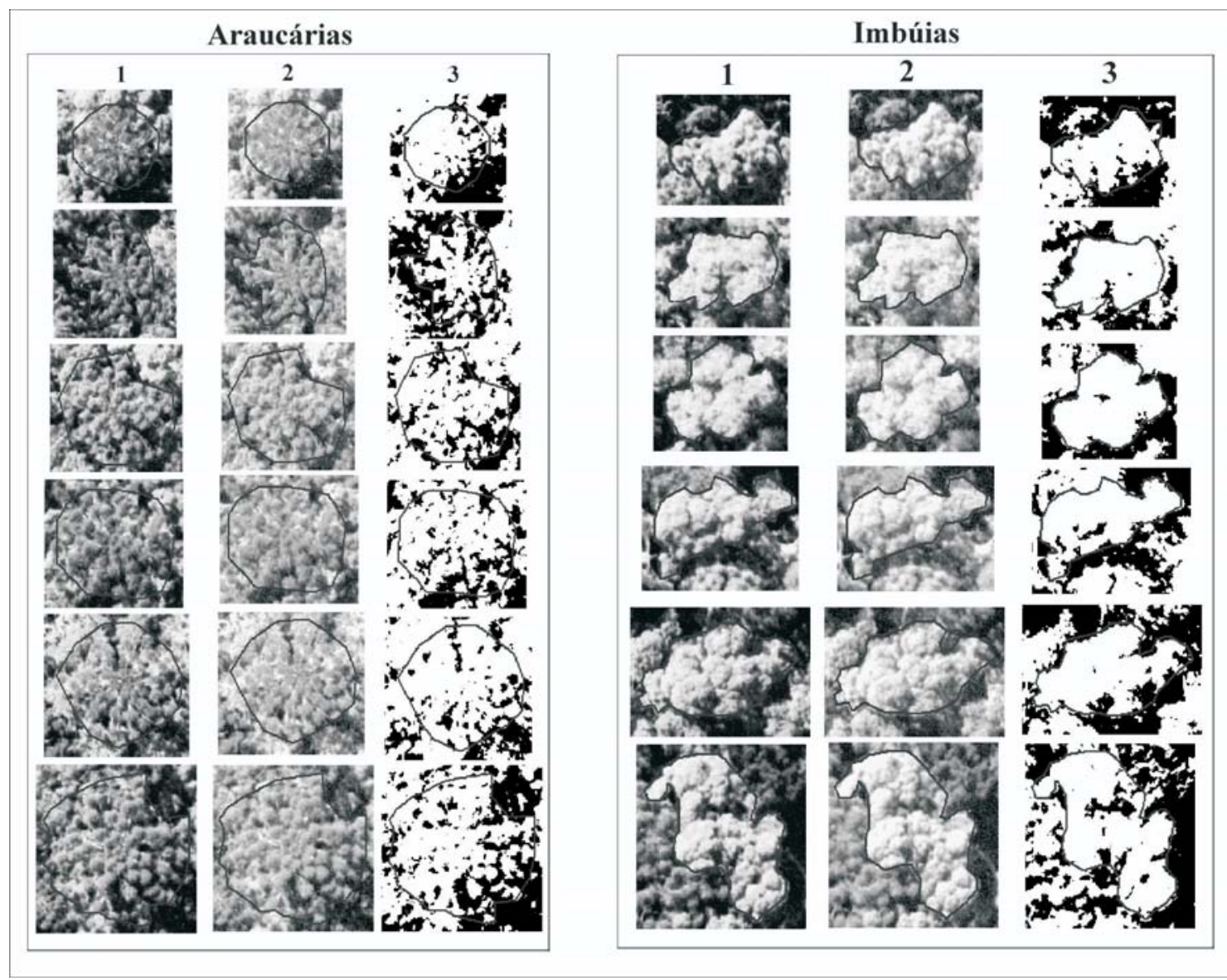

Figura 4 - Copas de araucária e imbúia na imagem colorida normal realçada (1), imagem iluminação/albedo (2) e imagem binária (3).

Figure 4-Individual crowns of araucária and Imbúia on enhanced normal color aerial photography (1), illumination/ albedo image (2) and bynary image (3). 


\section{REFERÊNCIAS BIBLIOGRÁFICAS}

BRANDTBERG, T. Remote sensing for forestry application - a historical retrospect. 2001. 9 p. www.dai.ed.ac/uk/CVonline/ LOCAL_COPIES/BRANDTBERG/UK.html

DISPERATI, A. A. Biodiversidade e sustentabilidade da Floresta Ombrófila Mista na Flona de Irati, PR. Irati: Unicentro, 2002a. 76p. (Relatório final do projeto do CNPq: Fomento à Pesquisa/ Apoio a Projeto de Pesquisa/Edital CNPq-01/2000-Faixa B).

DISPERATI, J. Identificazione e delimitazione di chiome di specie forestali attraverso foto aeree a grande scala nella Foresta Ombrófila Mista Del Paraná (Brasile). Parma: Universita degli studi di Parma, Facolta di Scienze Matematiche, Fisiche e Naturali, Corso di Laurea in Scienze Naturali, 2002b, 64 p.

DISPERATI, A. A. et al. Delineação visual de copas de árvores em fotografias aéreas de alta resolução de uma área com vegetação natural de Araucária: resultados parciais. In: SEMINÁRIO DE ATUALIZAÇÃO EM SENSORIAMENTO REMOTO E SISTEMAS DE INFORMAÇÕES GEOGRÁFICAS APLICADOS À ENGENHARIA FLORESTAL, 5., 2002, Curitiba. Anais... Curitiba: 2002. p.241- 242.

DISPERATI, A. A.; OLIVEIRA-FILHO, P. C. Delineação automática de copas de árvores em imagens de alta resolução: potencialidades e limitações. In: SEMINÁRIO DE ATUALIZAÇÃO EM SENSORIAMENTO REMOTO E SISTEMAS DE INFORMAÇÕES GEOGRÁFICAS APLICADOS Á ENGENHARIA FLORESTAL, 5., 2002. Curitiba. Anais... Curitiba: 2002. p.81-88.

GOUGEON, F. A. Individual tree identification from high resolution MEIS images. In: INTERNATIONAL FORUM ON AIRBORNE MULTISPECTRAL SCANNING FOR FORESTRY AND MAPPING (WITH EMPHASIS ON MEIS). 1992, Quebec. Proceedings... Quebec: Forestry Canada, Petawawa National Forestry Institute, 1992. p.117-128. (Information Report PI-X-113).

R. Árvore, Viçosa-MG, v.29, n.2, p.195-202, 2005
GOUGEON, F. A. Automatic individual tree crown delineation using a valley-following algorithm and a rule-based system. In: INTERNATIONAL FORUM ON AUTOMATED INTERPRETATION OF HIGH SATIAL RESOLUTION DIGITAL IMAGERY FOR FORESTRY, 1999, Victoria. Proceedings... Victoria: Canadian Forest Service, 1999. p.11-23.

LECKIE, D. G. Application of airborne multispectral scanning to forest inventory mapping. In: INTERNATIONAL FORUM ON AIRBORNE MULTISPECTRAL SCANNING FOR FORESTRY AND MAPPING (WITH EMPHASIS ON MEIS).1992, Quebec. Proceedings... Quebec: Forestry Canada, Petawawa National Forestry Institute, 1992. p.86-93. (Information Report PI-X-113).

MARTINS, S. V. Dinâmica de clareiras: aplicações na silvicultura e no manejo de florestas nativas. Folha Florestal, n. 95, p.15-17, 2000.

OLIVEIRA, Y. M. M. Correlações entre parâmetros dendrométricos em Araucaria angustifolia, utilizando fotografias aéreas. 1980. 133f. Dissertação (Mestrado em Engenharia Florestal) Universidade Federal do Paraná, Curitiba, 1980.

POUCH, G. W.; CAMPAGNA, D. J. Hypersherical direction cosine transformation for separation of spectral and illumination information in digital data. Photogrammetric Engineering and Remote Sensing, v.56, n.4, p.475-479, 1990.

QUACKENBUSH, L. J.; HOPKINS, P.F.; KINN, G.J. Developing forestry products from high resolution aerial imagery. Photogrammetric Engineering and Remote Sensing, v.66, n.11, p.1337-1346, 2000.

STELLINGWERF, D. A. Applications of aerial volume tables and aspects of their construction. In: SYMPOSIUM IUFRO S 6.05. REMOTE SENSING INCLUDING AERIAL-PHOTOGRAPHY. 1973, Freiburg. Proceedings... Freiburg: Freiburg Universität, p. 211-228.

WARNER, T. A.; LEE, J. Y.; McGRAW, J. B. Delineation and identification of individual trees in the eastern deciduous forest. In: INTERNATIONAL FORUM ON AUTOMATED INTERPRETATION OF HIGH SPATIAL RESOLUTION DIGITALIMAGERY FOR FORESTRY, 1999, Victoria. Proceedings... Victoria: Canadian Forest Service, 1999. p.81-91. 